\title{
MicroRNA-128 inhibition attenuates myocardial ischemia/reperfusion injury-induced cardiomyocyte apoptosis by the targeted activation of peroxisome proliferator-activated receptor gamma
}

\author{
XIAO CONG ZENG, LANG LI, HONG WEN and QI BI \\ Department of Cardiology, The First Affiliated Hospital of Guangxi Medical University, \\ Nanning, Guangxi 530021, P.R. China
}

Received May 28, 2015; Accepted April 6, 2016

DOI: $10.3892 / \mathrm{mmr} .2016 .5208$

\begin{abstract}
The aim of the present study was to investigate the effects of microRNA (miR)-128 inhibition on the targeted activation of peroxisome proliferator-activated receptor gamma (PPARG) and on cardiomyocyte apoptosis induced by myocardial ischemia/reperfusion (I/R) injury. In vitro, the expression of PPARG was detected by reverse transcription-quantitative polymerase chain reaction and western blotting in neonatal rat ventricular myocytes (NRVMs) and HEK293 cells transfected with the mimics or inhibitors of miR-128 or control RNA. Luciferase reporter assays were used to identify whether PPARG is a direct target of miR-128. In vivo, miR-128 was knocked down via ear vein injection of antagomir-128 in a rabbit myocardial I/R injury model. Western blotting investigated the activation of Akt [phosphorylated (p)-Akt] and the expression of total-Akt, PPARG and myeloid leukemia cell differentiation protein-1 (Mcl-1) in the myocardium. Cardiomyocyte apoptosis was examined with transmission electron microscropy and terminal deoxynucleotidyl transferase dUTP nick end labeling staining. PPARG mRNA and protein were downregulated in NRVMs transfected with miR-128 mimics, but upregulated by antagomir-128 compared with control. This indicates that PPARG is a direct miR-128 target. Activation of Akt (p-Akt), Mcl-1 and PPARG expression in the myocardium were increased by miR-128 inhibition. Furthermore, miR-128 antagomirs significantly reduced apoptosis in hearts subjected to I/R injury, which was blocked by the PPARG inhibitor GW9662. In conclusion, miR-128 inhibition attenuated I/R injury-induced cardiomyocyte apoptosis by the targeted activation of PPARG signaling.
\end{abstract}

Correspondence to: Dr Lang Li, Department of Cardiology, The First Affiliated Hospital of Guangxi Medical University, 22 Shuangyong Road, Nanning, Guangxi 530021, P.R. China E-mail: aban829@163.com

Key words: antagomir, apoptosis, myocardial ischemia/reperfusion, PPARG, miR-128

\section{Introduction}

It is well known that reperfusion strategies, including primary percutaneous coronary interventions and thrombolytic therapy, are important therapeutic strategies for acute ST segment-elevated myocardial infarction $(1,2)$. However, restoration of the blood flow through the previously ischemic myocardium can result in reperfusion-associated myocardial dysfunction and cell death $(3,4)$. To date, it has been widely accepted that apoptosis serves a pivotal role in the progress of myocardial ischemia/reperfusion (I/R) injury $(5,6)$. Therefore, it is important to identify a target for the development of an effective treatment of cardiomyocyte apoptosis, and therefore for attenuating myocardial I/R injury.

Peroxisome proliferator-activated receptor gamma (PPARG) is a ligand-activated transcription factor of the nuclear hormone receptor superfamily, which abundant in adipose tissue (7). PPARs are a family of at least three nuclear receptors $(\alpha, \delta$, and $\gamma)$, which regulate genes involved in lipid metabolism, adipocyte differentiation and inflammation (8). Previous studies have indicated that PPARG activation is also pivotal for the regulation of a variety of pathophysiologic processes within the cardiovascular system $(9,10)$. In addition, it has been demonstrated that activation of PPARG attenuates apoptosis induced by hypoxia/reoxygenation in cardiomyocytes (11) or myocardial I/R injury (12). Activation of Akt by phosphatidylinositol-3-kinase (PI3K) leads to the phosphorylation of a variety of downstream targets, including pro-apoptotic proteins, transcription factors and other protein kinases, thereby regulating cell survival (13). Induced myeloid leukemia cell differentiation protein-1 (Mcl-1), which is upregulated in several human cancers, is an anti-apoptotic Bcl-2 family protein (14). Previous studies have indicated that activation of PPARG inhibits apoptosis in cardiomyocytes, which may be associated with the activation of Akt (11) and expression of Mcl-1 (12).

MicroRNAs (miRNAs) are small noncoding RNAs, comprised of $\sim 22$ nucleotides. miRNAs negatively regulate gene expression via either degrading the target mRNA or by direct translational inhibition (15). Several candidate miRNAs (miRs), such as miR-27a (16), miR-27b (17), miR-130a and 
miR-130b $(18,19)$, have been reported to be negative regulators for PPARG expression. In a preliminary study, candidate miRNAs that bind to PPARG were screened using a bioinformatics algorithm (TargetScan 6.2; http://www.targetscan.org), according to a previous study (20). The results of this screen identified a putative miR-128 binding sequence (ACUGUGA) in the 3'-untranslated region (3'-UTR) of PPARG mRNA. Therefore, it was hypothesized that miR-128 inhibition may attenuate myocardial I/R injury-induced cardiomyocyte apoptosis by the targeted activation of PPARG.

The present study aimed to confirm that PPARG is a direct target of miR-128 in the HEK293 cell line and in cultures of neonatal rat ventricular myocytes (NRVMs). Furthermore, the role of miR-128 inhibition in preventing cardiomyocyte apoptosis through its PPARG activation-mediated effect was investigated in a rabbit myocardial I/R injury model. Myocardial I/R injury was induced by a protocol of $60 \mathrm{~min}$ of left anterior descending coronary artery (LAD) ischemia followed by $6 \mathrm{~h}$ of reperfusion (21). The aim was to shed new light on the roles of miRNAs, in particular miR-128, in myocardial I/R injury-induced cardiomyocyte apoptosis.

\section{Materials and methods}

HEK293 cell culture and preparation of primary cultured neonatal cardiomyocytes. A total of 24 neonatal (1-3 day old) male Sprague-Dawley rats were purchased from the Center for Experimental Animals, Guangxi Medical University (Nanning, China). The rats were maintained under a 12-h light/dark cycle at $22 \pm 1^{\circ} \mathrm{C}$. Food and water were available ad libitum. NRVMs were isolated from the rats, as described previously (22). Briefly, following anesthetization with intraperitoneal ketamine (50 mg/kg; Phoenix Pharmaceuticals, Inc., St. Joseph, MO, USA), the rats were sacrificed by decapitation and their hearts were aseptically removed. Ventricle tissues were dissected, minced and trypsinized at $37^{\circ} \mathrm{C}$ for $10 \mathrm{~min}$. Dispersed NRVMs were plated in 24-well plates in Dulbecco's modified Eagle's medium (DMEM; Invitrogen; Thermo Fisher Scientific, Inc., Waltham, MA, USA) containing $10 \%$ fetal bovine serum (FBS) and $0.1 \mathrm{mM}$ bromodeoxyuridine for further experiments. All procedures involving animal use were performed in accordance with the European Community Guidelines for the Care and Use of Animals, and the Institutional Ethics Committee for Animal Usage approved the research protocol. The HEK293 cell line was purchased from the Cell Resource Center (Shanghai Institutes for Biological Sciences, China Academy of Sciences, Shanghai, China). HEK293 cells were cultured in DMEM supplemented with $10 \% \mathrm{FBS}$ and $100 \mu \mathrm{g} / \mathrm{ml}$ penicillin/streptomycin in a humidified incubator at $37^{\circ} \mathrm{C}$ with $5 \% \mathrm{CO}_{2}$.

Transfection of NRVMs and HEK293 cells with miRNAs. The miRNAs were designed and chemically synthesized by Shanghai GenePharma Co., Ltd. (Shanghai, China). The following sequences were synthesized: Rat (rno)-miR-128 mimics, sense 5'-UCACAGUGA ACCGGUCUCUUU-3' and antisense 5'-AGAGACCGGUUCACUGUGAUU-3'; rno-miR-128 inhibitor (antagomir-128) 5'-AAAGAGACC GGTTCACTGTGA-3'; and negative control, sense 5'-UUC UCCGAACGUGUCACGUTT-3' and antisense 5'-ACGUGA
CACGUUCGGAGAATT-3'. NRVMs and HEK293 cells were transfected with the mimics or inhibitors of miR-128 or negative control RNA at a final concentration of $100 \mathrm{nM}$ using Lipofectamine 2000 (Invitrogen; Thermo Fisher Scientific, Inc.) and OPTI-MEM reduced serum medium (Thermo Fisher Scientific, Inc.), according to the manufacturer's instructions. Following transfection, the cells were incubated for 48 and $72 \mathrm{~h}$ prior to reverse transcription-quantitative polymerase chain reaction (RT-qPCR) and western blot analysis.

Luciferase reporter assay. The PPARG 3'-UTR containing the miR-128 target sequence (position 82-88) and mutant sequences (a single-base mutant in the 3'UTR) were chemically synthesized (GenScript, Nanjing, China) and cloned into the PGL3 control vector (Promega Corporation, Madison, WI, USA) downstream of the luciferase gene using the $X b a I$ site. The resulting plasmids were designated PPARG 3'-UTR-luci-wild type (WT) and PPARG 3'-UTR-luci-mutant (MUT). HEK293 cells were transfected with either PPARG 3'-UTR-luci-WT or PPARG 3'-UTR-luci-MUT in 24-well plates using Lipofectamine 2000 transfection reagent according to the manufacturer's instructions, and co-transfected with miR-128 mimic, antagomir-128 or the same concentration of negative control. Each well was also co-transfected with the pRL-TK plasmid (Promega Corporation) to determine the transfection efficiency. Subsequently, firefly and Renilla luciferase activity levels were measured in cells that were harvested $24 \mathrm{~h}$ post-transfection using the Dual-Luciferase Reporter Assay System (Promega Corporation). Each transfection was performed in triplicate.

Treatment groups and rabbit myocardial I/R injury model. A total of 40 healthy, adult male New Zealand white rabbits (weight, 2.0-2.5 kg; age, 8-12 weeks) were purchased from the Center for Experimental Animals at the Guangxi Medical University. Animals were housed at $25 \pm 2{ }^{\circ} \mathrm{C}$ and $60 \pm 5 \%$ humidity, and exposed to a 12:12 h light-dark cycle with pellet food and tap water ad libitum. The 40 rabbits were randomly divided into five groups $(\mathrm{n}=8 \mathrm{each})$. The sham-operated and I/R groups were administered vehicle only (10\% dimethylsulfoxide solution). The GW9662 group received the PPARG antagonist GW9662 (Sigma-Aldrich, St. Louis, MO, USA) at a daily dose of $0.5 \mathrm{mg} / \mathrm{kg}$ body weight by gastric gavage for three days. The antagomir-128 group received antagomir-128 at a single dose of $80 \mathrm{mg} / \mathrm{kg}$ body weight through ear marginal vein injection for three days prior to regional ischemia $(23,24)$. The antagomir-128+GW9662 group was administered antagomir-128 (a single dose of $80 \mathrm{mg} / \mathrm{kg}$ at three days prior to regional ischemia) and GW9662 $\left(0.5 \mathrm{mg} \cdot \mathrm{kg}^{-1} \cdot \mathrm{d}^{-1}\right.$ for three days). All animals were anesthetized using $20 \%$ urethane via an ear marginal vein injection. Following midline thoracotomy, a 4-0 silk ligature was placed under the LAD. The ends of the suture were threaded through polyethylene tubing to form a snare. The ends of the suture were pulled tight and a hemostat was used to clamp the snare to occlude the coronary artery. Following $60 \mathrm{~min}$ of ischemia, the ligature was untied and the snare was loosened, allowing the ischemic myocardium to re-perfuse for $6 \mathrm{~h}$. Sham-operated animals were subjected to all of the previously described procedures, except ligation of the LAD. 
Tissue sampling. Rabbits received the same treatment as described above and were subjected to $60 \mathrm{~min}$ of ischemia and $6 \mathrm{~h}$ of reperfusion. At $6 \mathrm{~h}$, the coronary artery was re-ligated, and Evans blue dye $(1 \mathrm{mg} / \mathrm{kg})$, which stains non-viable cells, was injected into the right ventricle to identify the myocardial area at risk. All animals were anesthetized by injection of $20 \%$ urethane, $10 \%$ potassium chloride $(10 \mathrm{ml})$ into the ear marginal vein, resulting in cessation of the heart beat during diastole. Subsequently, cardioectomy was performed, and hearts were removed, sliced and stained with 2,3,5-triphenyl-tetrazolium-chloride (TTC) to differentiate infarcted (TTC unstained) from viable myocardium (TTC stained). The border zone was identified as Evans blue unstained and TTC stained. A $2 \mathrm{~mm}$ section of myocardium tissue from the border zone was fixed in $4 \%$ formalin solution and embedded in paraffin for terminal deoxynucleotidyl transferase dUTP nick end labeling (TUNEL) assays. The border zone was also used for determination of the level of miR-128 in cardiomyocytes by RT-qPCR. In addition, the border zone was frozen in liquid nitrogen and stored at $-80^{\circ} \mathrm{C}$ for western blot analysis. The border zone was cut into a sample of $\sim 1 \mathrm{~mm}^{3}$ of subendocardial tissue to examine the ultrastructure of cardiomyocyte apoptosis by electron microscopy.

RT-qPCR analysis. Total RNA, including miRNA, was extracted from NRVMs and rabbit cardiac ventricular myocytes using TRIzol reagent (Invitrogen; Themo Fisher Scientitific, Inc.), according to the manufacturer's protocol. Following isolation, total RNA was incubated with RQ1 RNase-free DNase (Promega Corporation) to remove contaminating DNA. Using the ABI Prism 7300 Sequence Detection System (Applied Biosystems; Thermo Fisher Scientific, Inc.), the mRNA levels of PPARG and miR-128 levels were examined by RT-qPCR, as reported previously (25). The commercial kits used in the RT-qPCR were the TaqMan ${ }^{\circledR}$ Universal PCR Master Mix (cat. no. 4304437; Applied Biosystems; Thermo Fisher Scientific, Inc.), TaqMan ${ }^{\circledR}$ Reverse Transcription Reagents (cat. no. N8080234; Applied Biosystems; Thermo Fisher Scientific, Inc.), TaqMan ${ }^{\circledR}$ MicroRNA Assays (cat. no. 4427975; Applied Biosystems; Thermo Fisher Scientific, Inc.) and the TaqMan ${ }^{\circledR}$ MicroRNA Reverse Transcription kit (cat. no. 4366596; Applied Biosystems; Thermo Fisher Scientific, Inc.). $\beta$-actin or U6 was used as an internal reference. The primer sequences were as follows: PPARG forward, 5'-GCGACATCGACC AACTGAAC-3' and reverse, 5'-ACGGAGCGAAACTGA CACC-3'; miR-128 forward, 5'-CGCGCTCACAGTGAA CCG-3' and reverse, 5'-GTGCAGGGTCCGAGGT-3'; $\beta$-actin forward, 5'-TGTGATGGTGGGAATGGGTCAGAA-3' and reverse, 5'-TGTGGTGCCAGATCTTCTCCATGT-3'; and U6 forward, 5'-GCTTCGGCAGCACATATACTAAAAT-3' and reverse, 5'-CGCTTCACGAATTTGCGTGTCAT-3' (Shanghai GenePharma Co., Ltd.). The PCR cycling conditions were as follows: $95^{\circ} \mathrm{C}$ for $10 \mathrm{~min}$, followed by 40 cycles of $95^{\circ} \mathrm{C}$ for $15 \mathrm{sec}$ and $60^{\circ} \mathrm{C}$ for $35 \mathrm{sec}$. Individual samples were run in triplicate, and each experiment was repeated a minimum of three times. Data analyses were performed using the $2^{-\Delta \Delta C \mathrm{C}}$ method for calculating relative gene expression levels (26).

Western blot analysis. Total protein from NRVMs was extracted with $1 \%$ radioimmuniprecipitation assay lysis buffer (Beyotime Institute of Biotechnology, Jiangsu, China) containing $1 \mathrm{mM}$ phenylmethanesulfonyl fluoride. The supernatants were collected, and protein concentration was determined using a Bicinchoninic Acid Protein Assay kit (Pierce Biotechnology, Inc., Rockford, IL, USA). Proteins $(80 \mu \mathrm{g})$ were resolved on $12 \%$ sodium dodecyl sulfate-polyacrylamide gel electrophoresis (SDS-PAGE) gels and transferred to nitrocellulose membranes (Sigma-Aldrich). The membranes were blocked for $1 \mathrm{~h}$ at room temperature with $5 \%$ non-fat milk dissolved in PBS and incubated overnight at $4^{\circ} \mathrm{C}$ with rabbit anti-PPARG (1:500; cat. no. sc-7196) and anti- $\beta$-actin $(1: 1,000$; cat. no. sc-130656) polyclonal antibodies (both Santa Cruz Biotechnology, Inc., Dallas, TX, USA), followed by three 10-min washes with Tris-buffered saline solution containing $0.05 \%$ Tween-20 (TBST) and incubation with horseradish peroxidase-conjugated goat anti-rabbit immunoglobulin G (1:5,000; cat. no. sc-2004; Santa Cruz Biotechnology, Inc.). Subsequently, the membrane was washed three times for $10 \mathrm{~min}$ in TBST, and the protein was detected by chemiluminescence with the Pierce Enhanced Chemiluminescence Western Blotting Substrate (Pierce Biotechnology, Inc.).

The expression of phosphorylated Akt (p-Akt), total-Akt (t-Akt), PPARG and Mcl-1 protein in the border zone of the myocardium was examined by western blotting. Total protein was extracted from the border zone using standard procedures described previously (27). Proteins were separated by SDS-PAGE (as described for NRVM extracts) and incubated overnight at $4^{\circ} \mathrm{C}$ with rabbit anti-Akt (1:500; cat. no. sc-8312), anti-p-Akt (1:500; cat. no. sc-33437), anti-PPARG (1:500), anti-Mcl-1 (1:1,000; cat. no. sc-819) and anti- $\beta$-actin $(1: 1,000)$ polyclonal antibodies (all Santa Cruz Biotechnology, Inc.). Proteins were detected and visualized as described for NRVM extracts. All western blot data were quantified using Image J 1.48 software (National Institutes of Health, Bethesda, MD, USA) and data were normalized to $\beta$-actin.

Apoptotic cardiomyocyte ultrastructure and the TUNEL assay. The ultrastructure of apoptotic cardiomyocytes in the subendocardial tissue of the border zone of hearts subjected to the I/R protocol was examined by transmission electron microscopy (H-500; Hitachi, Tokyo, Japan), as described above. The tissues were prepared for transmission electron microscopy as described previously (28).

Myocardial apoptosis in the border zone was quantified by a TUNEL apoptosis assay kit (Nanjing KeyGen Biotech, Co., Ltd., Nanjing, China). All procedures were performed according to the manufacturer's instructions. Cells were defined as apoptotic if the whole nuclear area of the cell was labeled positively. TUNEL-positive myocytes were determined by randomly counting 10 fields using an inverted microscope (TE-300; Nikon Corporation, Tokyo, Japan). The apoptotic index (AI) was then determined as the number of apoptotic myocytes/total number of myocytes counted x 100 .

Statistical analyses. Data are presented as the mean \pm standard deviation. Statistical differences were determined using one-way analysis of variance followed by Fisher's Least Significant Difference tests. $\mathrm{P}<0.05$ was considered to indicate a statistically significant difference. All analyses 

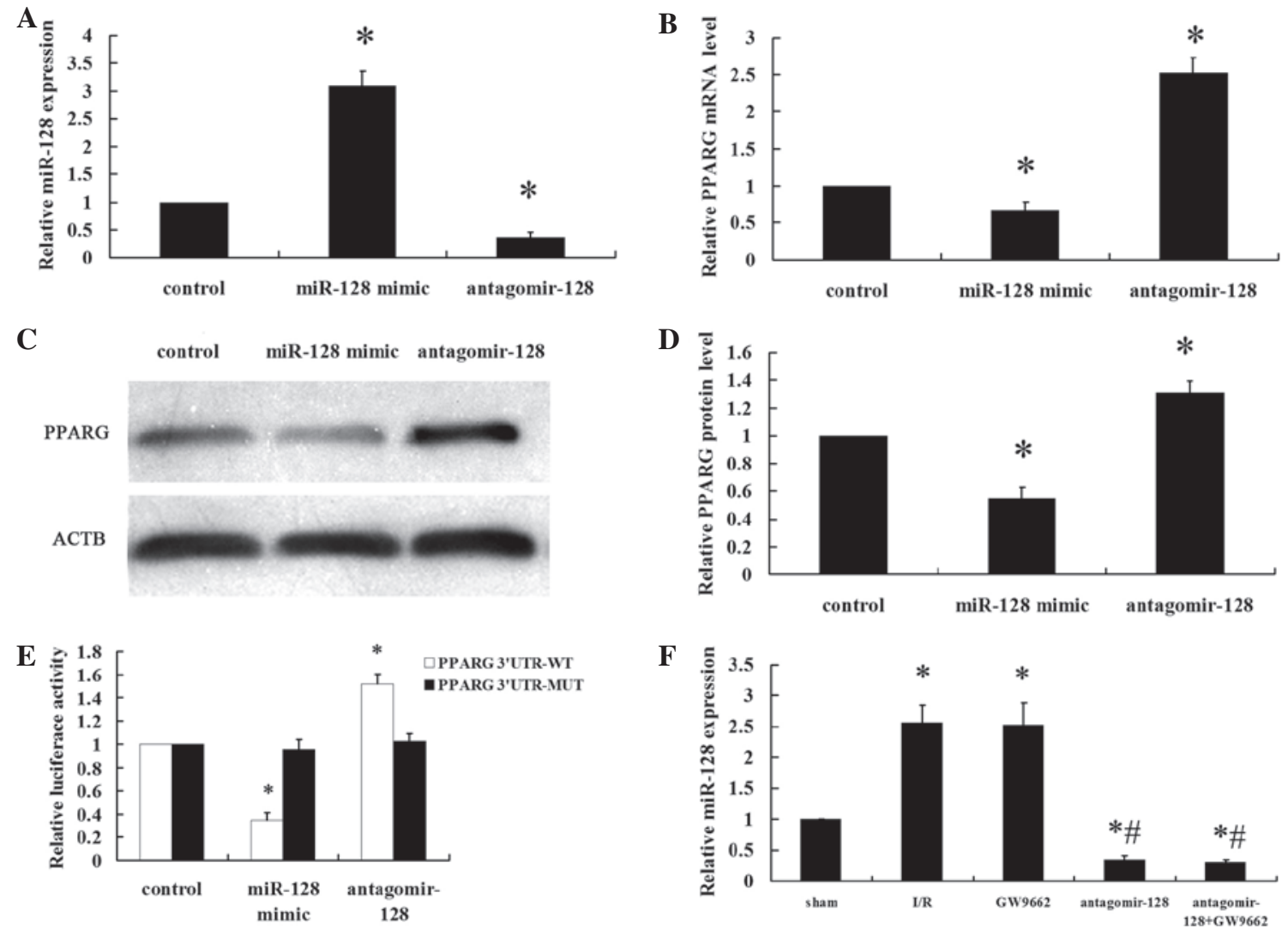

Figure 1. PPARG is a direct target gene of miR-128. Neonatal rat ventricular myocytes were transfected with a miR-128 mimic (100 nM), antagomir-128 $(100 \mathrm{nM})$ or miR-control (100 nM) for $48 \mathrm{~h}$. (A) The miR-128 levels were determined by RT-qPCR. (B) RT-qPCR showed that regulation of miR-128 levels affects the mRNA level of PPARG. (C) Representative western blot image of PPARG and ACTB protein levels. (D) Quantitative analyses of the expression of PPARG protein in the western blot analysis. PPARG values were normalized to ACTB expression levels. (E) Transient transfection of HEK293 cells with the PPARG 3'-UTR-luci-WT and PPARG 3'-UTR-luci-MUT constructs, along with the miR-128 mimic, antagomir-128 or miR-control added to the cultures $24 \mathrm{~h}$ following transfection. Luciferase activities were measured and normalized to the control luciferase activity. (F) The myocardial miR-128 levels were determined by RT-qPCR. Values are presented as the mean \pm standard deviation; $\mathrm{n}=8$ for each group. ${ }^{*} \mathrm{P}<0.05$ vs. the control group or sham group; ${ }^{\sharp} \mathrm{P}<0.05$ vs. the I/R group. PPARG, peroxisome proliferator-activated receptor gamma; miR, microRNA; RT-qPCR, reverser transcription-quantitative polymerase chain reaction; ACTB, $\beta$-actin; UTR, untranslated region; luci, luciferase; WT, wild type; MUT, mutant.

were performed using SPSS software, version 16 (SPSS, Inc., Chicago, IL, USA).

\section{Results}

PPARG gene is a target of miR-128. NRVMs were transfected with a miR-128 mimic $(100 \mathrm{nM})$, antagomir-128 $(100 \mathrm{nM})$ or miR-control $(100 \mathrm{nM})$ for $48 \mathrm{~h}$. The efficiency of transfection was measured by RT-qPCR (Fig. 1A). The miR-128 levels were significantly higher in the miR-128 mimic group compared with the levels in the control group $(\mathrm{P}<0.001)$. By contrast, the miR-128 levels were significantly lower in the antagomir-128 group compared with the control and miR-128 mimic groups $(\mathrm{P}<0.001)$. PPARG mRNA and protein expression levels were significantly downregulated in NRVMs that were transfected with the miR-128 mimic compared with the control group (P<0.001; Fig. 1B-D). By contrast, antagomir-128 significantly upregulated PPARG mRNA and protein levels compared with the control and miR-128 mimic groups $(\mathrm{P}<0.001)$. Data from the luciferase reporter assay showed that the downregulation of miR-128 following transfection with antagomir-128 resulted in a marked increase in luciferase activity of PPARG 3'-UTR-luci-WT compared with the control group $(\mathrm{P}<0.001)$, without obvious alterations in the luciferase activity of
PPARG 3'-UTR-luci-MUT compared with the control group (Fig. 1E). By contrast, the luciferase activity of PPARG 3'-UTR-luci-WT was reduced upon transfection of miR-128 mimics compared with the control group $(\mathrm{P}<0.001$; Fig. 1E). The myocardial miR-128 levels in the I/R and GW9662 groups were higher compared with the sham-operated group $(\mathrm{P}<0.001)$. Furthermore, the levels of myocardial miR-128 in the antagomir-128 and antagomir-128+GW9662 groups were significantly lower than in the sham and $\mathrm{I} / \mathrm{R}$ groups $(\mathrm{P}<0.001$; Fig. 1F).

In vivo miR-128 inhibition increases the activation of Akt and the expression of PPARG and Mcl-1 protein in myocardial $I / R$ rabbits. To further evaluate the role of miR-128 in PPARG signaling in vivo, miR-128 was knocked down via a single ear marginal vein injection of antagomir-128 $(80 \mathrm{mg} / \mathrm{kg})$ in a rabbit myocardial I/R injury model. Alterations in the protein levels of PPARG and the downstream signaling molecules Mcl-1, t-Akt and p-Akt were assessed by western blot analysis (Fig. 2A and B) and densitometry (Fig.2C-F). There were no significant differences in t-Akt protein expression between the five experimental groups (Fig. 2A and C). However, when examining the phosphorylated, and therefore activated from, of Akt (p-Akt; Fig. 2A and D), and the expression of PPARG and 
A

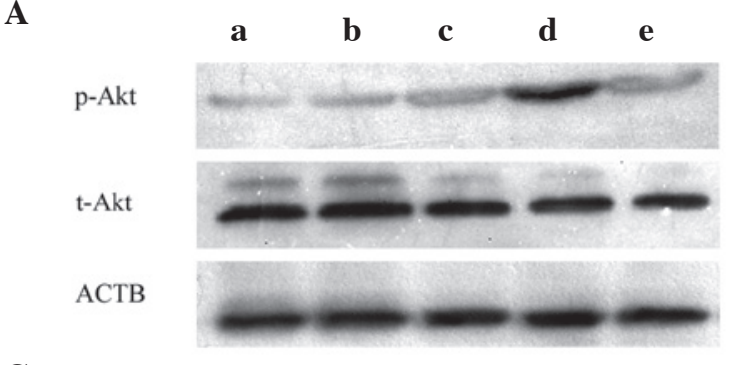

C

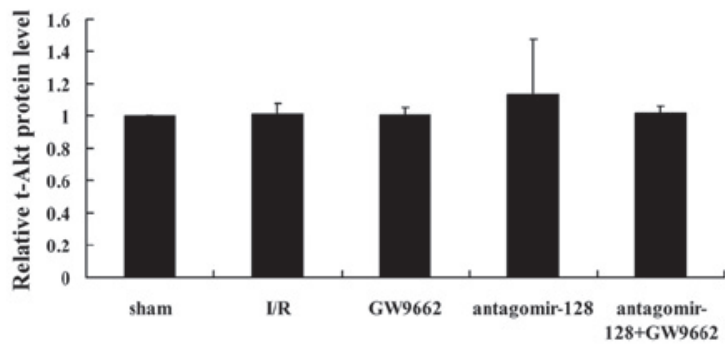

E

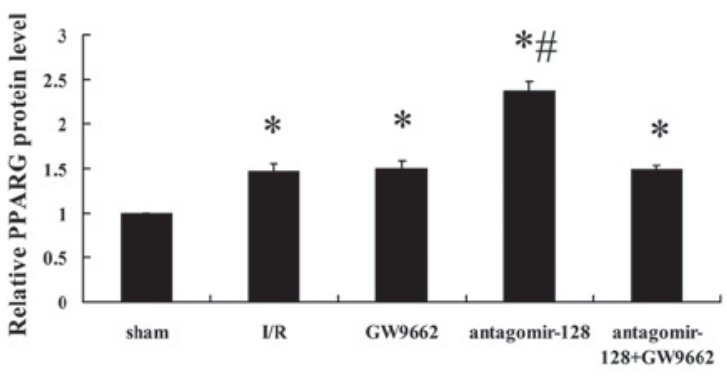

B

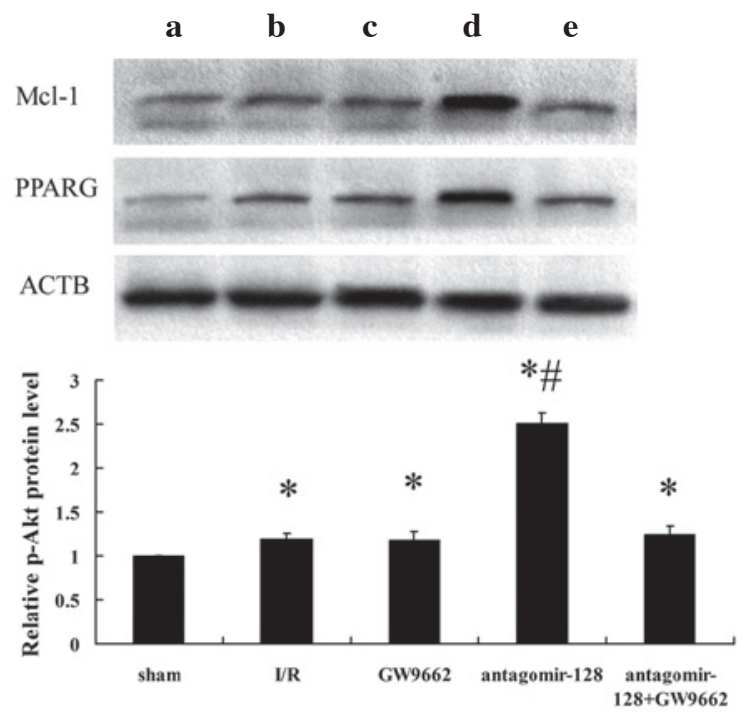

F

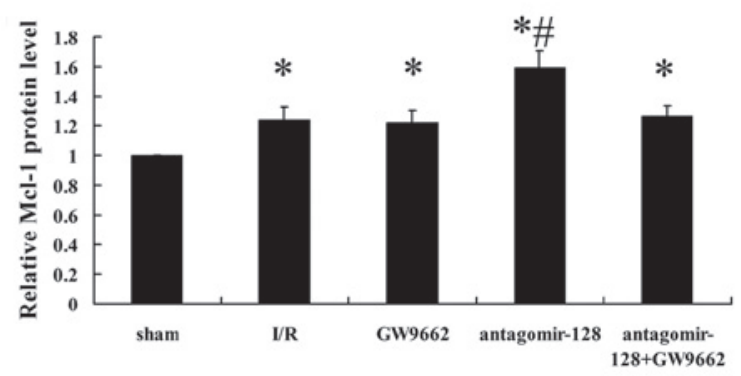

Figure 2. Determination of myocardial p-Akt, t-Akt, PPARG, Mcl-1 and ACTB protein expression by western blot analysis. (A) Representative western blot images showing p-Akt and t-Akt expression in extracts from the border zone of the myocardium of the (a) sham-operated, (b) myocardial I/R injury, (c) GW9662, (d) antagomir-128 and (e) antagomir-128+GW9662 group. (B) Representative western blot images showing PPARG and Mcl-1 expression in extracts from the border zone of the myocardium of the (a) sham-operated, (b) myocardial I/R injury, (c) GW9662, (d) antagomir-128 and (e) antagomir-128+GW9662 group. Quantification of the relative expression levels of (C) t-Akt, (D) p-Akt, (E) PPARG protein and (F) Mcl-1 protein. The values obtained by densitometric measurements were normalized to ACTB expression levels. Values are presented as the mean \pm standard deviation; $\mathrm{n}=8$ for each group. ${ }^{*} \mathrm{P}<0.05$ vs. the sham-operated group; ${ }^{\#} \mathrm{P}<0.05$ vs. the I/R group. p-Akt, phosphorylated Akt; t-Akt, total Akt; PPARG, peroxisome proliferator-activated receptor gamma; Mcl-1, myeloid leukemia cell differentiation protein-1; ACTB, $\beta$-actin; $\mathrm{I} / \mathrm{R}$, ischemia/reperfusion.

Mcl-1 (Fig. 2B, E and F), protein expression in all treatment groups was higher compared with the sham-operated group $(\mathrm{P}<0.001)$. Furthermore, the levels of p-Akt (Fig. 2A and D) and the expression of PPARG and Mcl-1 protein (Fig. 2B, $\mathrm{E}$ and $\mathrm{F}$ ) in the antagomir-128 group were significantly higher than in the I/R group $(\mathrm{P}<0.001)$. By contrast, there were no significant differences in $\mathrm{p}$-Akt and the expression of PPARG and Mcl-1 protein between the I/R, GW9662 and antagomir-128+GW9662 groups (Fig. 2D-F).

In vivo miR-128 inhibition protects against myocardial $I / R$-induced cardiomyocyte apoptosis. Subsequently, it was examined whether miR-128 inhibition was able to reduce cardiomyocyte apoptosis in vivo. Ultramicroscopic examination using transmission electron microscopy showed intact nucleoli, homogeneous chromatin and visible microvilli in the sham-operated group (Fig. 3A). In the I/R, GW9662, and antagomir-128+GW9662 groups, morphological features typical for apoptotic cell death, including pyknotic nuclei, heterochromatic clumping, peripheral condensation and margination of nuclear chromatin, were observed. The pyknotic nucleus assumed the appearance of a half-moon or crescent shape and the formation of apoptotic bodies was observed (Fig. 3B, C and E). In the antagomir-128 group, the peripheral chromatin condensation and aggregation of nuclear chromatin in dense masses was attenuated, while the phenomenon of crescent formation and apoptotic bodies was not observed (Fig. 3D). Furthermore, TUNEL assays indicated that the AI in the I/R, GW9662, antagomir-128, and antagomir-128+GW9662 groups were higher when compared with the sham-operated group $(\mathrm{P}<0.001$; Fig. 4A and $\mathrm{B})$. The $\mathrm{AI}$ in the antagomir-128 group was significantly lower than that in the $I / R$ group $(P<0.001)$. There was no significant difference in AI between the I/R, GW9662 and antagomir-128+GW9662 groups (Fig. 4B).

\section{Discussion}

The main findings of the current study are that PPARG mRNA and protein expression levels are downregulated in NRVMs transfected with miR-128 mimics compared with the control group. By contrast, the miR-128 inhibitor markedly upregulates PPARG mRNA and protein levels. Second, PPARG is a direct miR-128 target, as indicated by the luciferase reporter assays using PPARG 3'-UTRs containing the putative miR-128 binding site and a mutant version. Finally, an in vivo myocardial $\mathrm{I} / \mathrm{R}$ injury model in rabbits demonstrated that the intravenous injection of antagomir-128 increases the levels of p-Akt, Mcl-1 and PPARG in the myocardium compared with controls and 

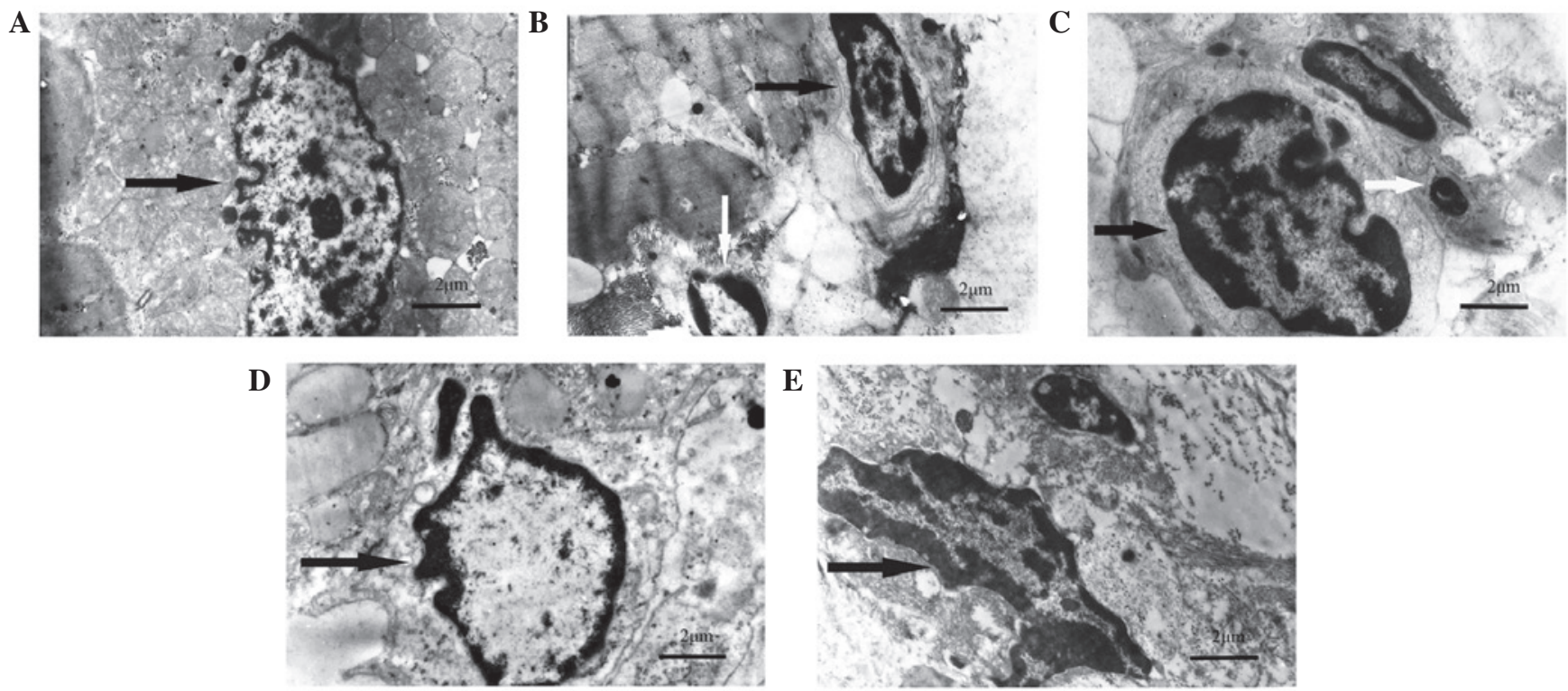

Figure 3. Transmission electron micrographs of the ultrastructural alterations of cardiomyocyte apoptosis in myocardial I/R injury experimental groups. (A) The sham-operated group shows intact nucleoli, homogeneous chromatin and visible microvilli (black arrow). (B) In the I/R group, heterochromatic clumping, peripheral condensation and margination of nuclear chromatin (black arrow) are observed; the pyknotic nucleus assumes the appearance of a half-moon or crescent shape (white arrow). (C) The GW9662 group exhibits heterochromatic clumping, peripheral condensation and margination of nuclear chromatin (black arrow), together with the formation of apoptotic bodies (white arrow). (D) In the antagomir-128 group, the peripheral chromatin condensation and aggregation of nuclear chromatin in dense masses was attenuated (black arrow). (E) The antagomir-128+GW9662 group shows heterochromatic clumping, peripheral condensation and margination of nuclear chromatin (black arrow). Magnification, $\mathrm{x} 7700$; scale bar $=2 \mu \mathrm{m}$. I/R, ischemia/reperfusion.

A
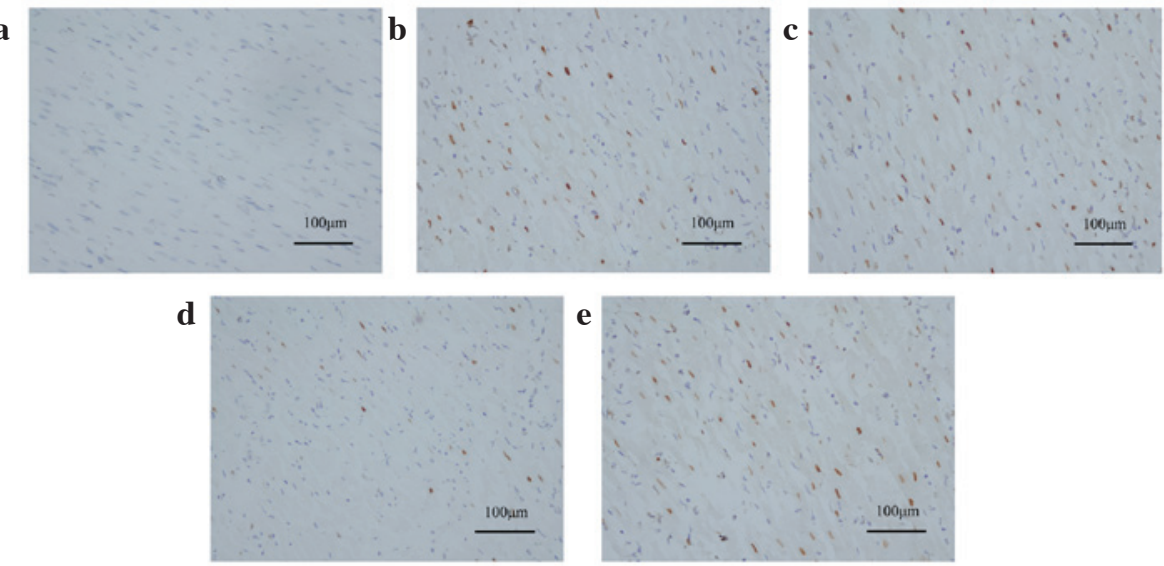

$\mathbf{B}$

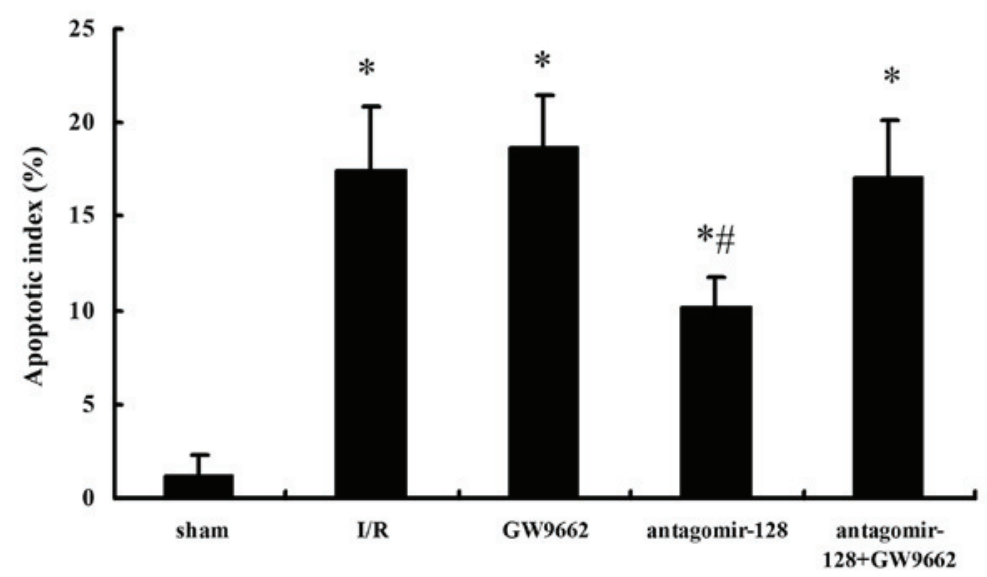

Figure 4. Assessment of myocardial apoptosis by TUNEL staining in the border zone of the myocardium in the myocardial I/R injury experimental groups. (A) Micrographs show myocardial apoptosis in the border zone as revealed by TUNEL staining. Cells were defined as apoptotic if the whole nuclear area of the cell was TUNEL-positive: (a) Sham-operated group; (b) I/R group; (c) GW9662 group; (d) antagomir-128 group; and (e) antagomir-128+GW9662 group. Magnification, $\mathrm{x} 400$; scale bar $=100 \mu \mathrm{m}$. (B) Quantitative analyses of the apoptotic index of myocardial I/R injury experimental groups. Values are presented as the mean \pm standard deviation; $n=8$ for each group. ${ }^{*} \mathrm{P}<0.05$ vs. the sham-operated group; ${ }^{*} \mathrm{P}<0.05$ vs. the I/R group. TUNEL, terminal deoxynucleotidyl transferase dUTP nick end labeling; I/R, ischemia/reperfusion. 
results in a significant reduction in apoptosis. This effect can be blocked by the PPARG inhibitor, GW9662. These results show for the first time, to the best of our knowledge, an anti-apoptotic effect of miR-128 inhibition in myocardial I/R injury, which is mediated through its effect on PPARG protein levels and the activation of signaling cascades downstream of PPARG, including activated Akt and the anti-apoptotic Bcl-2 family protein Mcl-1.

miRNAs are endogenous regulators of gene expression. Considering that cardiomyocyte apoptosis is a key cellular event in ischemic hearts that depends on gene expression (6), it is reasonable to hypothesize that miRNAs may be involved in I/R-induced cardiac apoptosis. It should be noted that several studies have demonstrated that miRNAs protect against cardiomyocyte apoptosis. For example, Li et al (29) reported that miR-145 protects cardiomyocytes against hydrogen peroxide-induced apoptosis through targeting the mitochondrial apoptotic pathway. Wang et al (30) reported that transfection of a lentivirus expressing miR-146a attenuated I/R-induced myocardial apoptosis and caspase-3/7 and -8 activities. Ye et al (12) reported that antagomirs against miR-29a or -29c significantly reduced myocardial infarct size and apoptosis in hearts subjected to IR injury.

A previous study indicated that PPARG activation attenuates apoptosis induced by myocardial I/R injury (12). By using computational approaches (TargetScan), the current study predicted a putative miR-128 binding sequence in the PPARG mRNA. To validate that PPARG is a miR-128 target, it was demonstrated that transfection with miR-128 mimics or inhibitors affects PPARG mRNA and protein levels in NRVMs. Subsequently, it was verified that PPARG is a direct miR-128 target using luciferase reporter assays in HEK293 cells. These results indicate that miR-128 directly modulates PPARG expression by binding to the 3'-UTR of PPARG and, thus, confirm that PPARG is a novel target of miR-128s.

To evaluate the biological role of miR-128 in PPARG signaling in vivo, miR-128 expression was knocked down via a single ear marginal vein injection of antagomir-128 in a rabbit myocardial I/R injury model. Activation of the PI3K/Akt pathway has been previously reported to prevent cardiomyocyte apoptosis and protect the heart from myocardial I/R injury $(31,32)$. Endothelial nitric oxide synthase is activated by Akt, which leads to nitric oxide production $(13,33)$. Furthermore, the activation of the PI3K/Akt pathway can mediate survival signals through the upregulation of members of the Bcl-2 family of anti-apoptotic proteins (34). For instance, a critical role of the Bcl-2 family member Mcl-1 in cardiomyocytes is to prevent the induction of cell death $(35,36)$. Previous studies have suggested that the activation of PPARG inhibits apoptosis in cardiomyocytes by increasing phosphorylation of Akt (11) and protein levels of Mcl-1 (12). In the present study, histological examination of the myocardium in the myocardial I/R injury model revealed that miR-128 inhibition was able to reduce cardiomyocyte apoptosis in vivo. Furthermore, the administration of antagomir-128 was observed to significantly increase the levels of p-Akt, Mcl-1 and PPARG in the myocardium, suggesting that miR-128 inhibition activates the PPARG signaling pathway in vivo. In response to the miR-128-modulated increase in PPARG protein, Akt was phosphorylated and Mcl-1 protein levels were increased, in turn attenuating I/R-induced cardiomyocyte apoptosis. Furthermore, antagomir-128 together with the PPARG inhibitor GW9662 significantly reduced p-Akt and Mcl-1 levels by antagonizing PPARG activation, thus resulting in significant reduction in the miR-128 inhibition-induced protective effects on cardiomyocyte apoptosis. These data show that activated PPARG is necessary for the anti-apoptotic effects in the myocardial $\mathrm{I} / \mathrm{R}$ injury model.

These data indicate that miR-128 inhibition may be a promising intervention for the management of apoptosis induced by myocardial I/R injury. Although the current studies were performed in an animal model and the experimental results cannot be extrapolated directly to humans, these results may provide a starting point for further preclinical studies to investigate whether targeting miR-128 is a valid therapeutic approach for cardioprotection following myocardial ischemia.

However, some limitations of the present study should be considered. First, the miR-128 mimics and inhibitors were derived from the rat sequence, while the in vivo investigations used adult rabbit hearts. However, computational miRNA target prediction analysis indicates that the miR-128 fragment 5'-UCACAGU-3' pairs well with the fragment 5'-ACUGUGA-3' of the PPARG 3' UTR, which is a highly conserved in mammals (e.g. rat, human, chimpanzee, rhesus, bushbaby, treeshrew, mouse and rabbit). Therefore, the difference in animal species for miR-128 mimics and inhibitors are unlikely to reduce the validity of the data interpretations. Second, additional immuno-stainings for anti-apoptotic proteins, apoptosis detection by flow cytometry and cardiac functional data, including echocardiography, will enable confirmation of the current finding in future studies.

Taken together, the results from the current study suggest that miR-128 inhibition has a protective effect against cardiomyocyte apoptosis during myocardial I/R, through the targeted activation of PPARG.

\section{Acknowledgements}

The present study was supported by the National Natural Science Foundation of China (grant no. 81560067) and the Youth Science Foundation of Guangxi Medical University (grant no. GXMUYSF2014028).

\section{References}

1. Canadian Cardiovascular Society, American Academy of Family Physicians, American College of Cardiology; American Heart Association, Antman EM, Hand M, Armstrong PW, Bates ER, Green LA, Halasyamani LK, et al: 2007 focused update of the ACC/AHA 2004 guidelines for the management of patients with ST-elevation myocardial infarction: A report of the American college of cardiology/American heart association task force on practice guidelines. J Am Coll Cardiol 51: 210-247, 2008.

2. Stenestrand U, Lindbäck J and Wallentin L; RIKS-HIA Registry: Long-term outcome of primary percutaneous coronary intervention vs. prehospital and in-hospital thrombolysis for patients with ST-elevation myocardial infarction. JAMA 296: 1749-1756, 2006.

3. Buja LM: Myocardial ischemia and reperfusion injury. Cardiovasc Pathol 14: 170-175, 2005.

4. Takemura G, Nakagawa M, Kanamori H, Minatoguchi S and Fujiwara $\mathrm{H}$ : Benefits of reperfusion beyond infarct size limitation. Cardiovasc Res 83: 269-276, 2009. 
5. Baines CP: How and when do myocytes die during ischemia and reperfusion: The late phase. J Cardiovasc Pharmacol Ther 16: 239-243, 2011.

6. Eefting F, Rensing B, Wigman J, Pannekoek WJ, Liu WM, Cramer MJ, Lips DJ and Doevendans PA: Role of apoptosis in reperfusion injury. Cardiovasc Res 61: 414-426, 2004.

7. Jozkowicz A, Dulak J, Piatkowska E, Placha W and Dembinska-Kiec A: Ligands of peroxisome proliferator-activated receptor-gamma increase the generation of vascular endothelial growth factor in vascular smooth muscle cells and in macrophages. Acta Biochim Pol 47: 1147-1157, 2000

8. Mersmann J, Tran N, Zacharowski PA, Grotemeyer D and Zacharowski K: Rosiglitazone is cardioprotective in a murine model of myocardial I/R. Shock 30: 64-68, 2008.

9. Goyal S, Arora S, Bhatt TK, Das P, Sharma A, Kumari S and Arya DS: Modulation of PPAR-gamma by telmisartan protects the heart against myocardial infarction in experimental diabetes Chem Biol Interact 185: 271-280, 2010.

10. Yasuda S, Kobayashi H, Iwasa M, Kawamura I, Sumi S, Narentuoya B, Yamaki T, Ushikoshi H, Nishigaki K, Nagashima K, et al: Antidiabetic drug pioglitazone protects the heart via activation of PPAR-gamma receptors, PI3-kinase, Akt and eNOS pathway in a rabbit model of myocardial infarction. Am J Physiol Heart Circ Physiol 296: H1558-H1565, 2009.

11. Kilter H, Werner M, Roggia C, Reil JC, Schäfers HJ, Kintscher U and Böhm M: The PPAR-gamma agonist rosiglitazone facilitates Akt rephosphorylation and inhibits apoptosis in cardiomyocytes during hypoxia/reoxygenation. Diabetes Obes Metab 11: 1060-1067, 2009

12. Ye Y, Hu Z, Lin Y, Zhang C and Perez-Polo JR: Downregulation of microRNA-29 by antisense inhibitors and a PPAR-gamma agonist protects against myocardial ischaemia-reperfusion injury. Cardiovasc Res 87: 535-544, 2010.

13. Ou HC, Lee WJ, Lee SD, Huang CY, Chiu TH, Tsai KL, Hsu WC and Sheu WH: Ellagic acid protects endothelial cells from oxidized low-density lipoprotein-induced apoptosis by modulating the PI3K/Akt/eNOS pathway. Toxicol App Pharmacol 248: 134-143, 2010

14. Mott JL, Kobayashi S, Bronk SF and Gores GJ: mir-29 regulates Mcl-1 protein expression and apoptosis. Oncogene 26: 6133-6140, 2007.

15. Hullinger TG, Montgomery RL, Seto AG, Dickinson BA, Semus HM, Lynch JM, Dalby CM, Robinson K, Stack C, Latimer PA, et al: Inhibition of miR-15 protects against cardiac ischemic injury. Circ Res 110: 71-81, 2012.

16. Lin Q, Gao Z, Alarcon RM, Ye J and Yun Z: A role of miR-27 in the regulation of adipogenesis. FEBS J 276: 2348-2358, 2009.

17. Wang J, Song Y, Zhang Y, Xiao H, Sun Q, Hou N, Guo S, Wang Y, Fan K, Zhan D, et al: Cardiomyocyte overexpression of miR-27b induces cardiac hypertrophy and dysfunction in mice. Cell Res 22: 516-527, 2012.

18. Lee EK, Lee MJ, Abdelmohsen K, Kim W, Kim MM, Srikantan S, Martindale JL, Hutchison ER, Kim HH, Marasa BS, et al: miR-130 suppresses adipogenesis by inhibiting peroxisome proliferator-activated receptor gamma expression. Mol Cell Biol 31: 626-638, 2011.

19. Pan S, Yang X, Jia Y, Li R and Zhao R: Microvesicle-shuttled miR-130b reduces fat deposition in recipient primary cultured porcine adipocytes by inhibiting PPAR-g expression. J Cell Physiol 229: 631-639, 2014

20. Lewis BP, Burge CB and Bartel DP: Conserved seed pairing, often flanked by adenosines, indicates that thousands of human genes are microRNA targets. Cell 120: 15-20, 2005.
21. Zeng XC, Li XS and Wen H: Telmisartan protects against microvascular dysfunction during myocardial ischemia/reperfusion injury by activation of peroxisome proliferator-activated receptor $\gamma$. BMC Cardiovasc Disord 13: 39, 2013.

22. Maass AH and Buvoli M: Cardiomyocyte preparation, culture and gene transfer. Methods Mol Biol 366: 321-330, 2007.

23. Krützfeldt J, Rajewsky N, Braich R, Rajeev KG, Tuschl T, Manoharan M and Stoffel M: Silencing of microRNAs in vivo with 'antagomirs'. Nature 438: 685-689, 2005.

24. Ren XP, Wu J, Wang X, Sartor MA, Qian J, Jones K, Nicolaou P, Pritchard TJ and Fan GC: MicroRNA-320 is involved in the regulation of cardiac ischemia/reperfusion injury by targeting heat-shock protein 20. Circulation 119: 2357-2366, 2009.

25. Herrera BM, Lockstone HE, Taylor JM, Wills QF, Kaisaki PJ, Barrett A, Camps C, Fernandez C, Ragoussis J, Gauguier D, et al: MicroRNA-125a is over-expressed in insulin target tissues in a spontaneous rat model of Type 2 diabetes. BMC Med Genomics 2: 54, 2009.

26. Schmittgen TD and Livak KJ: Analyzing real-time PCR data by the comparative C(T) method. Nat Protoc 3: 1101-1108, 2008

27. Shan H, Li X, Pan Z, Zhang L, Cai B, Zhang Y, Xu C, Chu W, Qiao G, Li B, et al: Tanshinone IIA protects against sudden cardiac death induced by lethal arrhythmias via repression of microRNA-1. Br J Pharmacol 158: 1227-1235, 2009.

28. Zhong JQ, Zhang W, Gao H, Li Y, Zhong M, Li D, Zhang C and Zhang Y: Changes in connexin 43, metalloproteinase and tissue inhibitor of metalloproteinase during tachycardia-induced cardiomyopathy in dogs. Eur J Heart Fail 9: 23-29, 2007.

29. Li R, Yan G, Li Q, Sun H, Hu Y, Sun J and Xu B: MicroRNA-145 protects cardiomyocytes against hydrogen peroxide $\left(\mathrm{H}_{2} \mathrm{O}_{2}\right)$-induced apoptosis through targeting the mitochondria apoptotic pathway. PLoS One 7: e44907, 2012.

30. Wang X, Ha T, Liu L, Zou J, Zhang X, Kalbfleisch J, Gao X, Williams D and Li C: Increased expression of microRNA-146a decreases myocardial ischaemia/reperfusion injury. Cardiovasc Res 97: 432-442, 2013

31. Liu H, Guo X, Chu Y and Lu S: Heart protective effects and mechanism of quercetin preconditioning on anti-myocardial ischemia reperfusion (IR) injuries in rats. Gene 545: 149-155, 2014.

32. Ren-an Q, Juan L, Chuyuan L, Wenjuan F, Chunyan H, Xuemei Y, Lin $\mathrm{H}$ and Hong N: Study of the protective mechanisms of compound Danshen tablet (Fufang Danshen Pian) against myocardial ischemia/reperfusion injury via the Akt-eNOS signaling pathway in rats. J Ethnopharmacol 156: 190-198, 2014.

33. Fulton D, Gratton JP, McCabe TJ, Fontana J, Fujio Y, Walsh K, Franke TF, Papapetropoulos A and Sessa WC: Regulation of endothelium-derived nitric oxide production by the protein kinase Akt. Nature 399: 597-601, 1999.

34. Limaye V, Li X, Hahn C, Xia P, Berndt MC, Vadas MA and Gamble JR: Sphingosine kinase-1 enhances endothelial cell survival through a PECAM-1-dependent activation of PI-3K/Akt and regulation of Bcl-2 family members. Blood 105: 3169-3177, 2005.

35. Thomas RL, Roberts DJ, Kubli DA, Lee Y, Quinsay MN, Owens JB, Fischer KM, Sussman MA, Miyamoto S and Gustafsson ÅB: Loss of MCL-1 leads to impaired autophagy and rapid development of heart failure. Genes Dev 27: 1365-1377, 2013.

36. Wang X, Bathina M, Lynch J, Koss B, Calabrese C, Frase S, Schuetz JD, Rehg JE and Opferman JT: Deletion of MCL-1 causes lethal cardiac failure and mitochondrial dysfunction. Genes Dev 27: 1351-1364, 2013. 